\title{
The Search for Gravitational Waves
}

\author{
F. J. Raab, \\ Reporting for the LIGO Scientific Collaboration \\ LIGO Hanford Observatory \\ Richland, WA, 99354, USA \\ Fred.Raab@ligo.org
}

\begin{abstract}
There has been a significant advance in the sensitivity of broadband searches for gravitational waves, using an international network of kilometer-scale laser interferometers. This network has recently concluded a 22-month search for gravitational waves, covering a frequency range from $50 \mathrm{~Hz}$ to several $\mathrm{kHz}$. Sensitivity to strains in space of the order of $10^{-21}$ brings a variety of potential signals into range, from distortions of spinning neutron stars in our galaxy to the mergers of neutron stars and black holes in the Virgo cluster of galaxies. Technology development for a second generation of interferometers has matured and construction of Advanced LIGO interferometers began in 2008 . This second generation of detectors, operating at the quantum limit, should provide a thousand-fold increase in the volume of space accessible to earthbound gravitational-wave detectors. Although the first detection of gravitational waves may be achieved by firstgeneration detectors before installation of Advanced LIGO begins in 2011, second-generation detectors will enable the routine detection of sources needed for gravitational-wave astronomy. This article describes the first and second generation detectors, gives some insight into what we have learned from observations and outlines future directions in this field.
\end{abstract}

\section{INTRODUCTION}

According to Special Relativity, information cannot travel through space faster than the speed of light. This requires that any field must have a wave phenomena associated with it. If an electric charge generating a static electric field is suddenly moved, its field at a distant point cannot change instantaneously. The field adjacent to the charge can change immediately, the field a short distance away can change after a short time, etc., until the field changes at the distant point at a time given by the distance divided by the speed of light. The rippling change in field traveling across space is the wave associated with this charge. For a mass generating a Newtonian gravity field the same applies; a gravitational wave must exist for gravity to be consistent with Special Relativity.

Newton (following Galileo's lead) showed that a body launched with some velocity into space follows a path independent of the nature of the object. Einstein expanded Newton's Principle of Equivalence to include massless objects, such as beams of light, described as a geometric phenomena. The General Relativity theory describes gravity as distortions in the fabric of space, consistent with Special

LIGO was constructed by the California Institute of Technology and Massachusetts Institute of Technology with funding from the National Science Foundation and operates under cooperative agreement PHY0107417. This paper has LIGO Document Number LIGO- P080061-02-Z.
Relativity, and the associated wave phenomenon is called a gravitational wave.

The curvature of space can be detected by simple surveying measurements. For instance the sum of the interior angles of any triangle drawn on a flat two-dimensional sheet will be 180 degrees; the same sum for a triangle drawn on the two-dimensional surface of a sphere will always be greater than 180 degrees. The surface of the sphere is said to have positive curvature.

Laser interferometer gravitational-wave detectors use freely suspended mirrors as survey markers for inertial frames in space [1], [2]. The spatial strain produced by a gravitational wave will cause motion of these frames that can be detected using laser light. This is illustrated schematically in Fig 1.
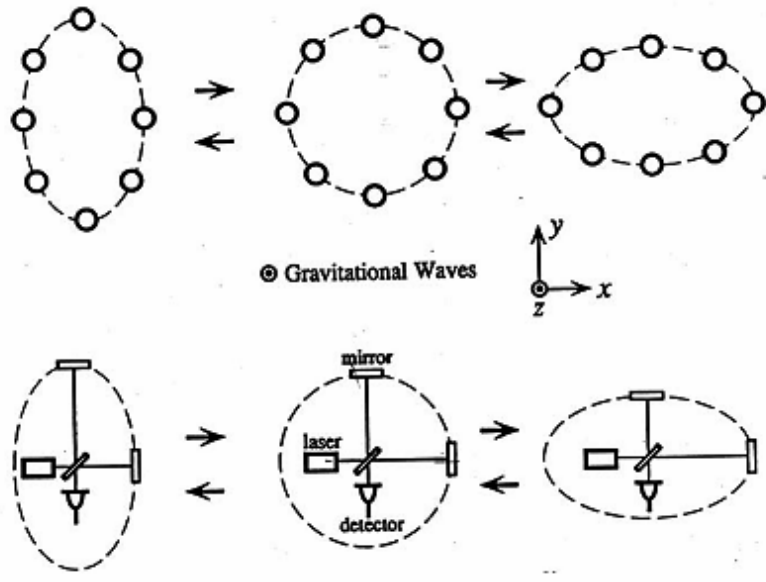

Figure 1. Schematic of gravitational wave strain and laser interferometer detector

Centered in the upper part of the figure, is a circle of points in space. To its left is shown the expected distortions of the circle when a gravitational wave, propagating vertically into the page with a particular polarization, reaches a maximum positive strain. To the right is the image when the gravitational wave reaches its maximum negative strain. The strain stretches one axis of space transverse to the propagating gravitational wave while simultaneously shrinking the perpendicular axis transverse to the wave. The strain amplitude $\mathrm{h}$ is the difference in the major and minor axes of the resulting ellipse, divided by the diameter of the original 
circle. The bottom part of the figure shows a schematic laser interferometer, with a beam-splitting mirror marking the center of the circle and two mirrors marking the edge of the circle, separated by 90 degrees. As space expands and contracts the free mirrors follow the points in space. Laser light transiting the interferometer picks up a phase shift depending on the relative lengths of the two arms, which is detected at the photodetector.

\section{REAL LASER INTERFEROMETERS}

The strains at Earth from astrophysical sources are expected to be of order or less than $10^{-21}$ RMS, a number comparable to the ratio of the thickness of a human hair to the 4 light-year distance between the Sun and Alpha Centauri. An interferometer with kilometer-scale arms thus requires a sensitivity to differential mirror displacements of $\sim 10^{-18} \mathrm{~m}$ (or 1 millifermi) RMS. This corresponds to $\sim 10^{-10}$ radians of optical phase. Residual seismic motion transmitted to the mirrors and the Brownian motion of the mirror and suspension structures in equilibrium at room temperature provide the natural background for these devices. The fundamental noise in sensing the interference is due to shot noise arising from the quantum nature of light. The relative complexity of these devices offers many additional opportunities for noise to enter the system. In this section we use the LIGO detectors as an example. Many of these elements are incorporated into the other laser interferometers in the worldwide network.

\section{A. Facilities and Vacuum System}

The LIGO facilities consist of buildings that house experimental equipment connected by vacuum tubes that conduct the laser beams between buildings. A corner-station building and two end-station buildings form the two arms of the interferometer. At Hanford there is an additional midstation building centered on each arm to house the end mirrors of the 2-km interferometer. The arms have an opening angle of 90.00 degrees. The arms are configured to be straight and oriented as nearly transverse to Earth's gravitational acceleration as is reasonable, given Earth's curvature. The arms of the Hanford and Livingston facilities were aligned (modulo 90 degrees) to optimize sensitivity to the same polarization of gravitational wave within certain constraints imposed by the two sites. This allows optimal use of coincidence operation of the two interferometer sites to improve detection confidence.

The LIGO vacuum system consists of vacuum chambers that hold vibration-isolated optical platforms and the beam tubes along the arms. The evacuated chambers provide a quiet environment for the optics, eliminating acoustic coupling and reducing the Brownian motion of the mirrors from residual gas collisions to an acceptable value. The evacuated beam tube (see Fig. 2) reduces optical path length fluctuations due to residual gas and scattering from the beam-tube walls, which are not isolated from vibration. The beam tube provides a 1.2meter clear aperture to support multiple interferometer beams.

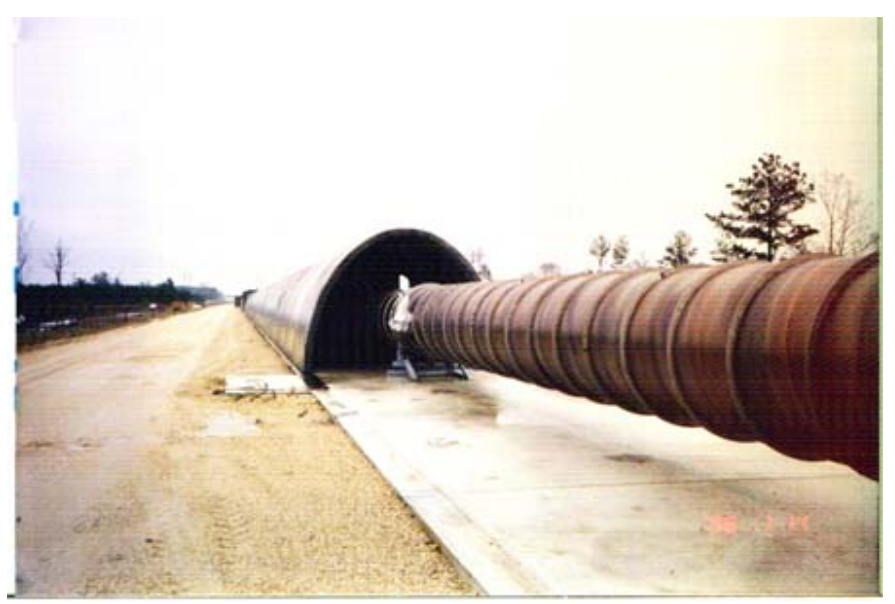

Figure 2. The 1.2-m-diameter LIGO beam tube, mounted on its foundation, with $15-\mathrm{cm}$ thick concrete covers.

The beam tubes operate at $10^{-9}$ Torr, principally limited by hydrogen outgassing from the steel, with much lower partial pressures of gases with higher polarizabilities. Because the hardware within the vacuum chambers is the major source of outgassing into the beam tubes, we concentrated pumping capacity near these chambers rather than distributing pumps along the beam tube. An extended bake-out of the completed vacuum tube was done and leaks in the $50 \mathrm{~km}$ of weld seams at each site were certified to be negligible. Each $2 \mathrm{~km}$ long tube segment was wrapped in a $15-\mathrm{cm}$ thick layer of fiberglass insulation and baked at temperatures of 150-160 C for 3-4 weeks. Large magnet power supplies, on loan from Fermi National Accelerator Laboratory, fed approximately 1800 A of current through the length of the tubing to provide heat, while cryopumps were used temporarily along the length of the tubing to remove evolved gases. Post-bake accumulation measurements certified that $\mathrm{H} 2$ outgassing was below $10^{-13}$ Torr $1 \mathrm{~cm}^{-2} \mathrm{~s}^{-1}$, while water vapor and other species outgassed at rates between $10^{-17}$ and $10^{-20}$ Torr $1 \mathrm{~cm}^{-2} \mathrm{~s}^{-1}$.

\section{B. Initial Interferometers}

The initial interferometer [3] (shown schematically in Fig. 3.) evolved from design principles successfully demonstrated with respect to minimizing displacement noise [4] and phase noise [5], and techniques for controlling laser frequency [6], [7], [8], length [9], [10], [11], [12], [13] and alignment [14], [15], [16], [17] in interferometer test beds. It uses a powerrecycled, Fabry-Perot-Michelson configuration [9], shown schematically in figure 3 .

A laser system delivers light to the input optics, where further conditioning and stabilization of the light occurs. A beam splitter divides the light equally between the two arms. A 4-km (or 2-km) Fabry-Perot cavity is formed in each arm by a mirror in the corner station and a mirror in the end (or mid-) station. The cavities increase the phase shift per unit strain for the light reflected from the arms. The mirrors are suspended from a modular cage using a single loop of fine wire, allowing the mirrors to respond freely to gravitational waves. Magnets glued to the mirrors and voice coils attached to the cage are used for fine actuation of the mirrors. The 
suspension cages are attached to passive seismic isolation stacks [18] inside the vacuum chamber as shown in Fig. 4.

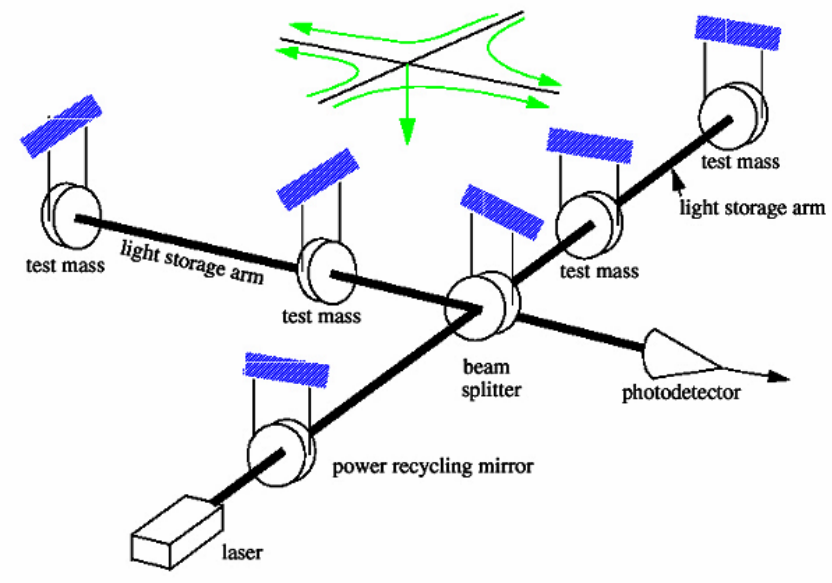

Figure 3. Schematic of a power-recycled Fabry-Perot-Michelson interferometer.

Load forces are brought out of the vacuum chambers through support rods and bellows to an actuation system for coarse positioning and alignment. The interferometer is operated in a balanced state, with light resonating in the arms and then returning toward the laser source after recombining at the beam splitter, so the other port is in relative darkness. A photo-detector in this 'dark port' detects any imbalance of the interferometer, but is insensitive to perturbations that affect both arms symmetrically. A heterodyne detection scheme is used for sensing the cavity lengths and alignments throughout the interferometer. This involves impressing phase-modulation sidebands on the light at radio frequencies (RF), which are converted into amplitude modulations at various photodiodes by position and alignment errors between the light and the cavities.
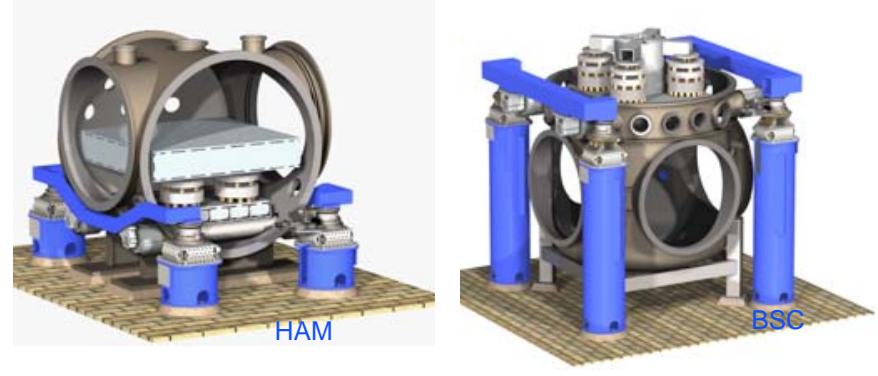

Figure 4. The vibration isolation systems for the two separate vacuum chambers.

A control system maintains the interferometer in this "balanced" state at all times. The effect of any gravitational wave is recorded on the control signals that maintain this condition. These control signals are calibrated by applying small, known forces to the mirrors that mimic the response to a gravitational wave. Since the interferometer in maintained in this balanced state by the control system, signals are recovered with relatively little light absorbed at the photo-detector, so most of the light is returned toward the laser. The light returning toward the laser is recycled [19], [20] by a powerrecycling mirror to increase light in the interferometer. Typically each photon is recycled approximately 50 times in this type of interferometer.

The sensitivity of gravitational-wave detectors [21], [1] is limited by fundamental and practical concerns as shown in Fig. 5. The vertical axis gives the noise-equivalent strain that would be obtained in a $1 \mathrm{~Hz}$ bandwidth and the horizontal axis gives the frequency of operation. At high frequencies, the quantum nature of light gives rise to shot noise on recombination of photons at the beam splitter [22], [23], [24]. This noise limits the smallest strain that can be sensed. Background motion of the mirrors (not due to gravitational waves) will limit sensitivity at lower frequencies. At the lowest frequencies there is a vibration background that arises due to seismic and man-made sources. This cannot be eliminated, since we rely on a solid connection to Earth to support our mirrors against Earth's gravity. Vibration is mitigated by cascaded low-pass mechanical filters, which give rise to a sharp seismic cut-off.

In the intermediate range of frequencies, the only remaining vibrations should be the cumulative effects of vibrations of the atoms comprising the suspended mirrors of the interferometer. These vibrations are in thermal equilibrium with the room-temperature environment of these components, so we expect an average energy of $k_{B} T$ per mode, where $k_{B}$ is Boltzmann's constant and $\mathrm{T}$ is the temperature. This "thermal noise" [25], can be separated into motions arising from the mirror substrate [26], [27], [28] and the suspension fibers [29], [30].

The same photon fluctuations that give rise to shot noise also cause an irreducible background of radiation pressure fluctuations on the mirrors. The interplay of shot noise and radiation pressure enforces the Heisenberg uncertainty principle for these macroscopic detectors. The sensitive region above the sum of these noise sources defines the signal band for gravitational-wave interferometers. The noise-equivalent strain at $150 \mathrm{~Hz}$ is approximately $3 \times 10^{-22}$.

There are also limits imposed by the observatory environment and facilities. The most fundamental limit is the Newtonian gravity noise of Earth [31], [32] (labeled gravitational gradient in Fig. 5), which sets the low-frequency bound for terrestrial detectors. Fluctuations in the column density of residual gas in the kilometer-scale beam tubes of these interferometers induce phase shifts on the light that mimic the effect of gravitational wave strains. Stringent requirements on the vacuum in these beam tubes mitigate this noise. Finally, the small clear aperture of these long beam tubes require baffling to prevent stray light from glancing reflections from the beam-tube walls which cannot be vibration isolated [33]. The efficacy of the baffling determines the "stray light" limit in Fig. 5. 


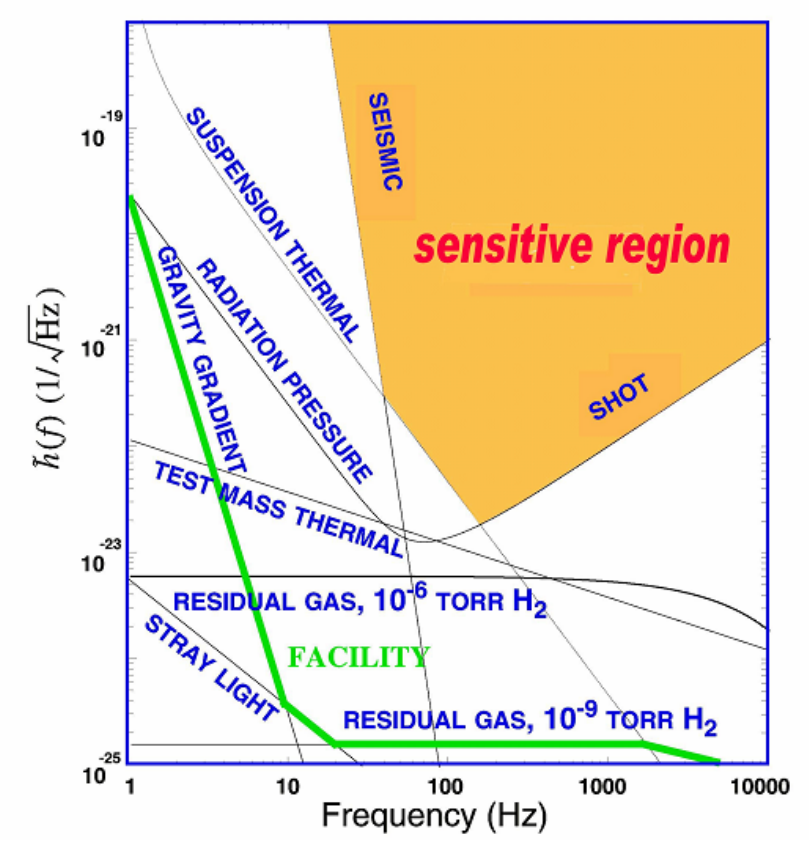

Figure 5. Noise sources that affect interferometer sensitivity.

\section{THE INTERNATIONAL DETECTOR NETWORK}

Kilometer-scale laser interferometers in North America, Europe and Asia have been operated as part of a worldwide detection network. In North America, the NSF-sponsored LIGO operates detectors (Fig. 6) at two facilities in the US incorporating interferometers in an L-shaped vacuum system with 4-km arm lengths. A 4-km interferometer (H1) and a 2$\mathrm{km}$ interferometer $(\mathrm{H} 2)$ share this vacuum at the Hanford, Washington facility. A single 4-km interferometer (L1) occupies a similar vacuum system at the Livingston, Louisiana facility. These facilities were designed to house a succession of interferometers of increasing sophistication and sensitivity as the critical technologies for these instruments evolve. The Initial LIGO interferometer was designed to have a plausible, if not probable, chance to make the first detections of gravitational waves, while a far more powerful detector, known as Advanced LIGO was under development. Advanced LIGO was intended to make gravitational-wave detections a common occurrence. Caltech and MIT operate the LIGO facilities and support labs on their campuses for the LIGO Scientific Collaboration. The collaboration, which includes approximately 600 scientists and engineers on four continents, sets the scientific agenda for LIGO, supporting operations, data analysis and technology development for future upgrades.

The GEO600 detector (Fig. 7) near Hannover, Germany [34] is operated by the GEO collaboration of scientists from Germany and the United Kingdom. It name derives from the 600-meter length of its arms. GEO personnel are members of the LIGO Scientific Collaboration as well. Because of its shorter arms, GEO600 is not expected to have comparable sensitivity to LIGO, but it has provided important experience with more advanced suspension and vibration-isolation technologies. GEO600 also uses a signal-recycling mirror at the gravitational wave detection port. This mirror causes gravitational wave signals on the light to resonate in the interferometer within a certain band of frequencies. Changing the reflectivity and position of this mirror allows the response of the interferometer to be tuned. One can choose to operate in a relatively broad band mode or as a narrowly tuned detector. Many of these features and all of this experience will be incorporated into Advanced LIGO interferometers.
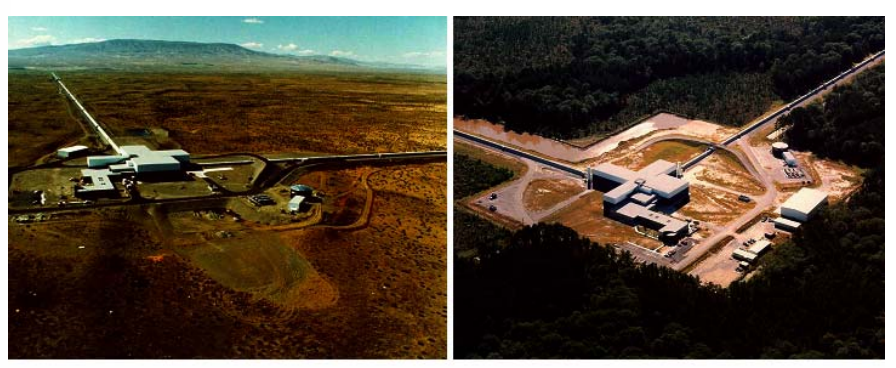

Figure 6. LIGO facilities in Washington (left) and Louisiana (right).

The VIRGO detector (Fig. 7) is a 3-km instrument [35] situated in Cascina (near Pisa), Italy, operated by a collaboration of scientists from Italy and France. VIRGO employs a similar configuration to the LIGO detectors, but has a more advanced vibration-isolation system. VIRGO sensitivity should eventually be comparable to the Initial LIGO detector over most of its frequency band, but it can operate at lower frequencies, comparable to the Advanced LIGO detector.
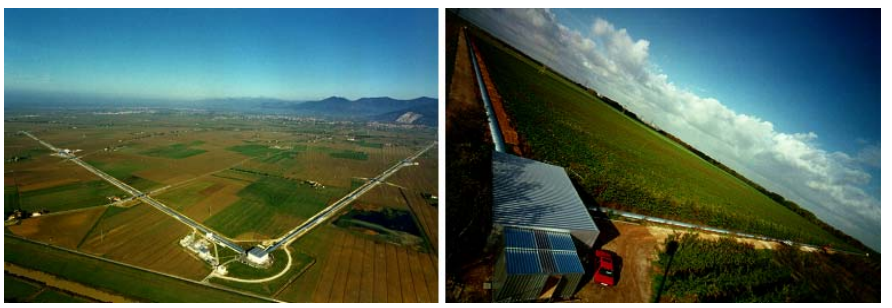

Figure 7. VIRGO (left) and GEO600 (right) detectors.

TAMA300 (Fig. 8) near Tokyo, Japan [36] has 300-meter arm lengths. The basic optical configuration is similar to that employed in the LIGO and VIRGO detectors. Because of its smaller size, TAMA300 cannot achieve the sensitivity of the larger interferometers, but it was the first of these large-scale detectors to come into operation.

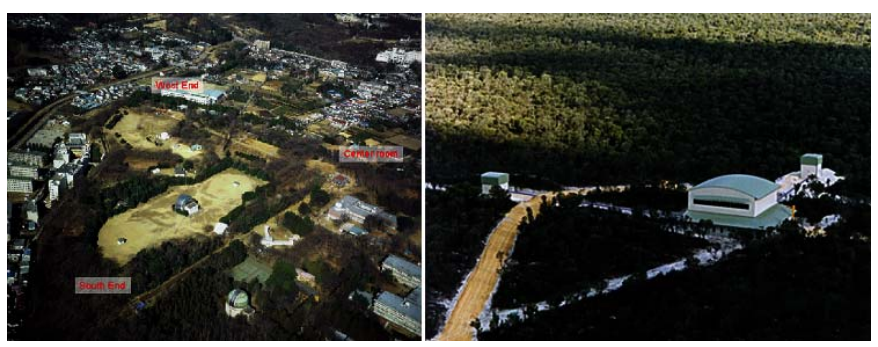

Figure 8. TAMA300 (left) and Gingin site (right). 
The Gingin site (Fig. 8) in Western Australia currently has a small interferometer being used to test Advanced LIGO optics. It is hoped that this site could eventually be expanded to house a $3-\mathrm{km}$ interferometer in the Southern Hemisphere.

\section{INITIAL LIGO DETECTOR OPERATIONS}

Observatory construction began with rough grading of the observatory sites in 1994 and 1995, followed by detail design of the facilities. Major facility construction began in 1996 and laboratory spaces became available for equipment in 1997 and 1998. Fig. 9 provides a timeline of detector commissioning following the inauguration of completed facilities in autumn, 1999.
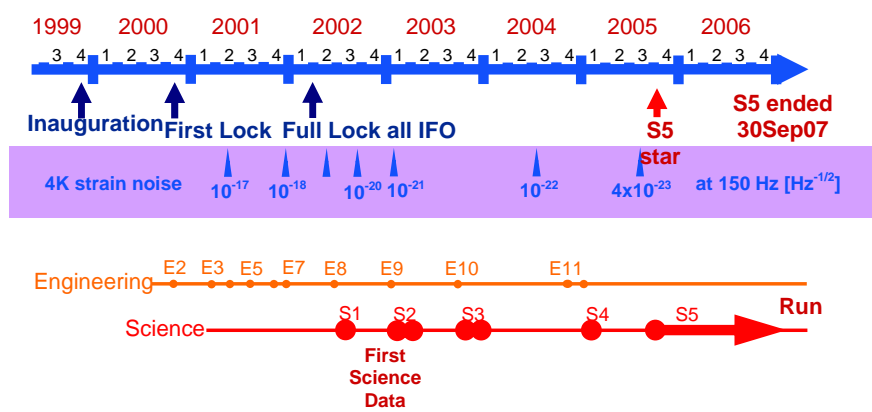

Figure 9. Timeline of LIGO commissioning and science runs.

The first laser light transited one of the evacuated arms for the Hanford $2 \mathrm{~km}$ interferometer in late November 1999. The first engineering run (E1), a $20 \mathrm{~h}$ data run utilizing a single locked arm, was taken during the first week of April 2000. This inaugurated a series of engineering runs used to test the interferometer under extended running conditions as the hardware and control systems were brought on line. The Hanford $2 \mathrm{~km}$, power-recycled, Fabry-Perot Michelson interferometer was locked for the first time by October 2000 and characterization of lock acquisition and robustness were begun. By early 2002, all interferometers had been operated in a power-recycled, Fabry-Perot Michelson configuration.

By the middle of 2002, interferometer sensitivity had been improved by three orders of magnitude above initial results as subsystem troubleshooting improved and the control system was implemented and tuned. Science runs were then conducted whenever dramatically new levels of sensitivity were achieved for LIGO's detectors. These runs were scheduled to last from a few weeks to a few months to identify if there were frequent events at the new sensitivity level before engaging in more exhaustive commissioning efforts to extend the range of the detectors.

The original target for the Initial LIGO detector was to achieve a noise-equivalent strain of $10^{-21}$ RMS in a $100-\mathrm{Hz}$ bandwidth at the frequency of peak sensitivity. Although this was achieved in the S3 run, the duty cycle of the detectors was impaired by man-made sources of noise, particularly logging near the Louisiana facility. An active seismic attenuation system, consisting of sensors and hydraulic actuators mounted external to the vacuum chambers [37], was installed at the Louisiana observatory during the long break between S3 and $\mathrm{S} 4$. This resulted in a threefold increase in observing duty cycle at Louisiana during the month-long S4 run, despite a several day interruption due to failure of the electrical power connection to the site. The most recent spectrum also shows a significant improvement in sensitivity at frequencies above $100 \mathrm{~Hz}$, as we increased the laser power injected into the interferometers to reduce the influence of shot noise. To handle larger laser powers it is important to match the wavefront of the light to the figures of the mirrors, overcoming both manufacturing tolerances and differences in heating of the mirrors by small amounts of absorbed laser power. A thermal compensation system [38] has been installed to allow adaptive tuning of the optical profiles of the mirrors by selectively applying heat from a $\mathrm{CO} 2$ laser to certain zones of the mirrors.

By summer of 2005, the initial LIGO interferometers had reached their design sensitivity (Fig. 10) and science run S5 was initiated in autumn with a goal of collecting one integrated year of triple coincidence data. In May 2007, the Virgo detector came on-line and joined the S5 run under a joint operating agreement between the LIGO Scientific Collaboration and Virgo Collaboration.

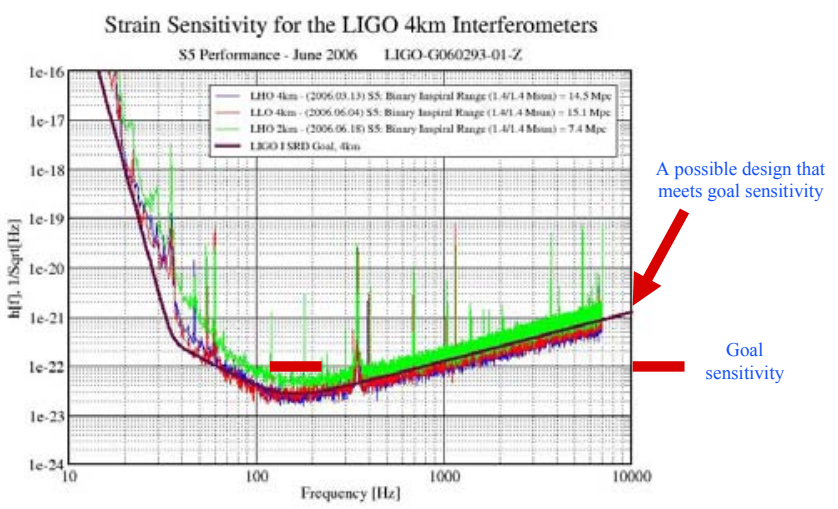

Figure 10. Sensitivity of LIGO interferometers during the S5.

\section{ASTROPHYSICAL SEARCHES}

The various laser interferometer groups have adopted a common data format to minimize technical barriers to combined data analysis. The most sensitive searches have been LIGO observing runs, with $\mathrm{S} 1$ through $\mathrm{S} 4$ conducted in coincidence with the laser interferometers GEO600, Virgo, TAMA300 and with the Allegro and AURIGA resonant-mass detectors. These runs were planned well in advance to coordinate the commissioning and observing schedules of these detectors as much as possible. By the time of the S5 run, the laser interferometers had well exceeded the sensitivity of the resonant-mass detectors. TAMA300 was off-line, commissioning new vibration isolation improvements.

We use the waveform for two inspiraling neutron stars, of 1.4 solar masses each, as a benchmark waveform to derive a figure of merit for these broad band detectors: the range to which we can typically make a high-confidence detection of such an event. (Neutron star inspirals were chosen because the 
waveform is well known and it extends across the broad band of detector response.) This inspiral range metric has improved steadily as the interferometer strain sensitivity has been increased and extended to lower frequencies. The best interferometer in S1 had a range covering the Milky-Way Galaxy and the Magellanic Clouds. By S2, the range included the Andromeda Galaxy (M31) at $\sim 1 \mathrm{Mpc}$ and by S3, the range covered a significant fraction of the distance to the Virgo supercluster. The S5 range was approximately $15 \mathrm{Mpc}$, which includes the Virgo cluster of galaxies.

The data have been searched by a variety of methods for gravitational waves. There are four qualitative categories of searches: for periodic sources, such a spinning neutron stars or strange-quark stars; for quasiperiodic, well-known waveforms, such as the inspiral of neutron stars or black holes; for bursts with poorly known waveforms, such as supernovae, gammaray burst progenitors, mergers of neutron-stars to form black holes and for cosmic-string-generated bursts; for stochastic signals of cosmological or astrophysical origin. These searches can be targeted (such as for pulsars or gamma-ray bursts detected by electromagnetic astronomy) or untargeted.

Unfortunately, we have not detected gravitational waves in data analyzed to date for these science runs. We have been able to publish more than 30 papers with improving observational upper limits [39] on a variety of gravitationalwave sources. Although there has been impressive progress in the range at which various sources could have been observed, the short duration of the first four science runs prevented very strong limits on the rates of gravitational wave generation from being obtained. The S5 analysis will probe for compact binary inspirals and coalescences over clusters of galaxies; probe for neutron star ellipticities on the $10^{-7} \mathrm{scale}$; and establish an observational limit on the cosmological background of audio-frequency gravitational waves comparable to or better than the estimated "big-bang nucleosynthesis" bound [40], [41].

Two recently released analysis results demonstrate that even null gravitational wave searches yield important astrophysical information at the current level of sensitivity.

GRB 070201 was a short gamma-ray burst detected by several satellites on Feb 1, 2007. The Interplanetary Network reported an error box for its location on the sky that overlapped the spiral arms of M31, the Andromeda Galaxy. The merger of a neutron star into another neutron star or black hole is a suggested mechanism for powering such short GRBs. However, another mechanism, called a short gamma repeater (SGR), is believe to be powered by a magnetic field reconnection event on a magnetar. These two mechanisms are expected to strongly differ in the energy radiated into gravitational waves. The two LIGO detectors at Hanford were on during this time, but analysis showed no detectable excess energy consistent with gravitational waves. From this we concluded that GRB070201 could not have been a merger in M31, although it could have been an SGR in M31 or a merger much farther away than M31 [42].

The Crab pulsar and its associated nebula, located 6,500 light years away in the constellation Taurus, was formed in a spectacular supernova explosion in $1054 \mathrm{CE}$. The Crab pulsar is the youngest known neutron star for which there is accurate timing data. Neutron stars are believed to have a semi-solid crust that gradually relaxes over time into a more symmetrical shape. The pulsar is known from radio observations to be to slowing down in rotation, mostly by regular deceleration with occasional glitches. We expect most of the energy released to be powering the nebula, but relatively small distortions could radiate significant power in gravitational waves. Assuming the distortion is locked to the rotation of the pulsar, the signal should appear at approximately $59.6 \mathrm{~Hz}$, which is twice the rotation rate of the pulsar. We searched for such a signal using the first 9 months of data from S5, prior to a large timing glitch, and found none [43]. This implies that the power being emitted in gravitational waves by the Crab pulsar is less than $4 \%$ of the rotational power loss. This is the first time that the "spindown limit" for emission of gravitational waves by any pulsar has been beaten by direct observation with a gravitational wave detector. It implies that the Crab pulsar is round to within a few meters, compared to the approximately $10-\mathrm{km}$ diameter of the neutron star.

\section{The NeXt Generation OF Detectors}

During the installation, commissioning and operation of the initial LIGO interferometers, a significant R\&D effort has been focused on developing designs and prototypes for a far more powerful detector, known as Advanced LIGO [44]. Advanced LIGO instrumentation will replace the initial LIGO hardware at the observatory facilities at Hanford and Livingston. Construction of Advanced LIGO was started in April 2008. Fig. 11 illustrates the Advanced LIGO features.

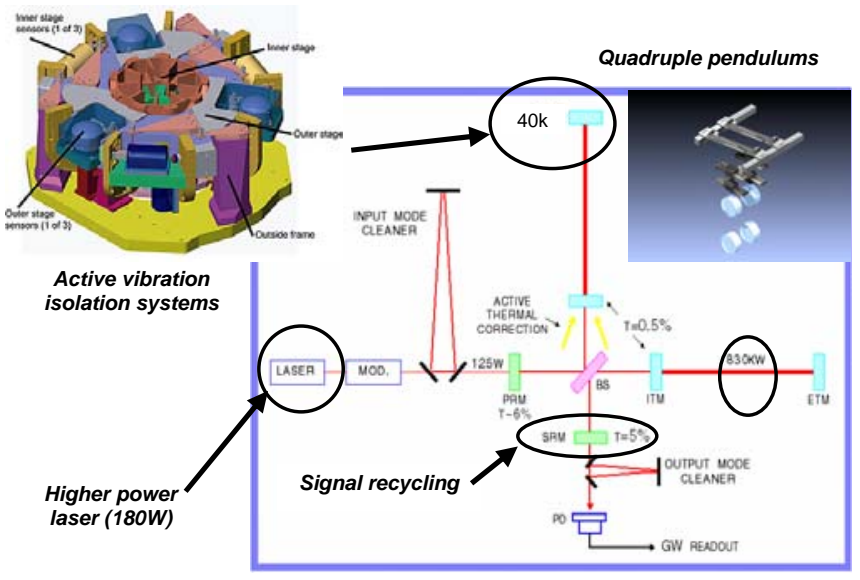

Figure 11. A schematic of the Advanced LIGO interferometer.

An active seismic isolation system and a multiple-layer quadruple suspension system [37] provide improved seismic isolation. The monolithic mirror assembly consists of a fusedsilica fiber welded to fused silica mirror and penultimate mass. This arrangement and improved coatings on the mirror result in a lower thermal noise design. The shot noise at high frequencies is improved by using a higher power laser and a homodyne detection scheme, using the output mode cleaner to block RF sidebands. The greater mass of the larger mirror reduces displacements from both thermal noise and from radiation pressure fluctuations. Finally a signal recycling 
mirror is added to the interferometer to allow its frequency response to be modified.

Fig. 12 shows the range of frequency responses available with this design, by changing either the transmission or position of the signal recycling mirror. Mode 0 corresponds to no signal recycling mirror (transmission $\mathrm{T}=100 \%$ ) and mode 1a corresponds to using a $20 \%$ transmission signal recycling mirror, both having 25 Watts of laser light incident on the interferometer. In mode $1 \mathrm{a}$ the minimum level of quantum noise is reduced but the frequency response is broadened by reducing quantum noise relative to mode 0 at lower and high frequencies. Mode $1 \mathrm{~b}$ is similar to mode $1 \mathrm{a}$, but with $125 \mathrm{~W}$ of incident laser power. Mode 2 corresponds to the conditions as mode $1 \mathrm{~b}$, except for a slight position displacement of the signal recycling mirror. The correlation of amplitude and phase fluctuations reflected from the output port becomes more apparent for this case as the double minima in the quantum noise. The mode 3 shows how quantum noise can be optimized for lower frequency operation by further repositioning the SRM and decreasing incident laser power to $20 \mathrm{~W}$. Finally, mode NB shows how a reduction of SRM transmission to $\mathrm{T}=1 \%$ deepens and narrows the quantum noise minima. This type of narrow-band tuning might be advantageous to improve sensitivity to periodic sources. The estimated background from thermal noise is shown in gray. From $20-2000 \mathrm{~Hz}$ the major contributor is from the mirror coatings; at lower frequencies, pendulum thermal noise becomes important. As we see from the figure, thermal noise reductions would be necessary to achieve the full benefit of manipulating the quantum noise at low frequencies.

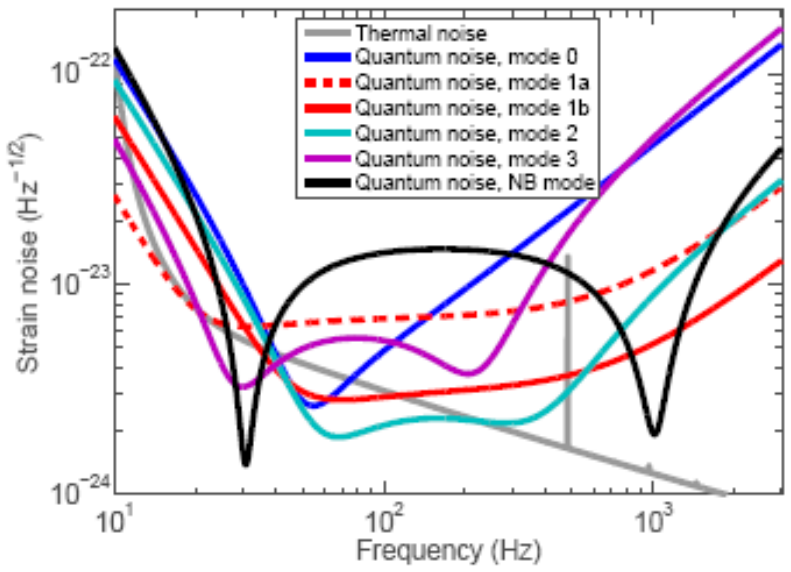

Figure 12. Different frequency responses for quantum noise by varying the laser power and the tuning of the signal recycling mirror in Advanced LIGO.

The various modes of operation are explained in the text. An estimate of thermal noise is given by the gray curve.

The signal band of Advanced LIGO will extend to lower frequencies $(\sim 10 \mathrm{~Hz})$ and have approximately ten times greater range for detecting sources of gravitational waves. This increases the probability of detection of order a thousandfold, ensuring that expected sources, such as compact binary inspirals and mergers, will be detected regularly.

Advanced LIGO designs have matured and some test components have become available. The $\mathrm{H} 1$ and L1 interferometers are currently being "enhanced" by installing these test systems and modifying the optical read-out system. This allows field tests of Advanced LIGO designs and techniques in the most realistic and sensitive operating conditions available as well as improving performance for a final science run (S6) while the full instrumentation for Advanced LIGO is being constructed. These changes include:

- Installing a $35 \mathrm{~W}$ laser, using one of the amplifier modules for the Advanced LIGO laser.

- Converting from a heterodyne to a homodyne detection scheme for the gravitational wave signal. Heterodyne techniques will conveniently provide alignment information for all mirrors and position information for the beam splitter and recycling mirrors. However, the sidebands, which do not resonate in the long arm cavities, are not filtered within the interferometer as well as the carrier and thus introduce phase and amplitude noise.

- Using an Output Mode Cleaner to transmit only the carrier light to the AS photodiode. The difference in arm lengths will then be locked with a small static offset.

- Supporting the suspended output mode cleaner with a single-stage active vibration isolation system intended for use in the input and output chambers in Advanced LIGO.

- Replacing materials used for voice coil actuator magnets and suspension cage earthquake stops to mitigate noise due to magnetic domain flipping and electric charging.

We expect these changes to increase the range of the detector by a factor of 2 to 2.5 , providing an approximately ten-fold increase in detection probability in the period before Advanced LIGO is ready.

\section{A GLIMPSE OF THE FUTURE}

Within the next 7-10 years, Advanced LIGO will be making regular detections of gravitational waves in the audio band of frequencies. One can think of this band, concentrated on the end points and of stellar evolution and its remnants, as the gravitational wave analogue to optical astronomy. Just as electromagnetic astronomy has been enriched by opening new frequency bands, there is a richness of sources to be studied at other frequencies in the gravitational-wave sky.

The study of super-massive black holes at the centers of galaxies and numerous other sources will require detectors that have good response in the milli-Hz range of frequencies. The joint ESA/NASA LISA mission [45\} will rise above Earth's noisy gravity (literally) with a triangular arrangement of spacecraft separated by distances of 5 million km. Fig. 13 compares the frequency responses of Advanced LIGO and LISA. 


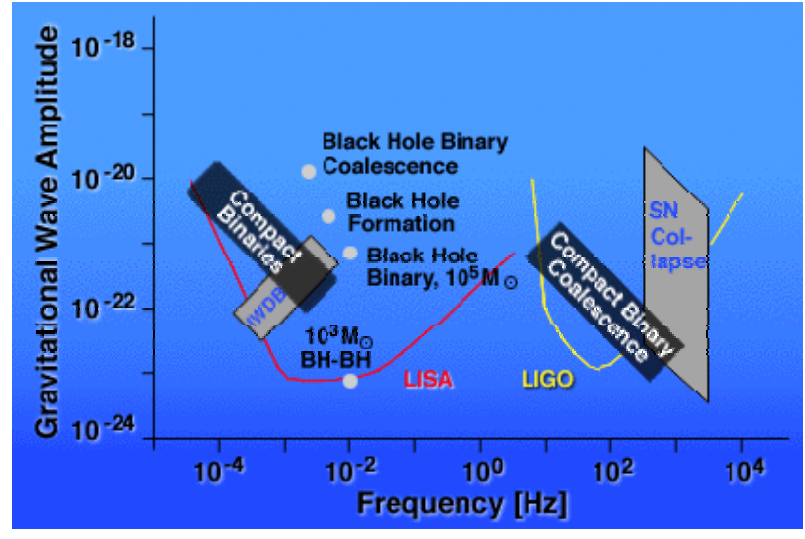

Figure 13. LISA and LIGO frequency bands and sources.

As Advanced LIGO construction proceeds, the LISA Pathfinder/ST-7 mission will demonstrate the core technologies needed for both LISA's interferometers and for the "drag-free" spacecraft control that protects LISA's proof masses from disturbance. LISA's progress will be subject to the success of LISA Pathfinder and subsequent funding decisions by ESA and NASA. We hope that the launch of the full LISA mission will occur within the next decade to open this exciting window on the gravitational sky.

LISA can be viewed as the analogue of a radio telescope in electromagnetic astronomy. One can see from Fig. 13 that there is not yet a planned detector to fill in the two orders of magnitude frequency gap between LIGO and LISA. The lowest frequency region of the gap will likely require the use of spacecraft. An option being studied for the $1-10 \mathrm{~Hz}$ region is to locate a LIGO-like third-generation detector underground to escape Newtonian gravity noise, which is most severe at Earth's surface. Preliminary calculations indicate that large reductions of gravity noise might be realized underground and detailed studies of what is possible in practice are now in the planning stages using existing and future underground science facilities. Of course, the design of such a facility will require substantial progress in reducing thermal noise and quantum noise at these lower frequencies.

\section{SUMMARY}

These are very exciting times in the field of gravitational wave detection. The first generation of kilometer-scale detectors on Earth's surface has broken barriers for sensitivity and robustness that some suspected might be unattainable only a few decades ago. These advances have expanded the distances over which the universe can be searched for gravitational wave sources from a few $\mathrm{kpc}$ to tens of Mpc. This is an enormous increase in the searchable volume of space and hence in detection probability. Newly developed technologies to increase this volume already are appearing in test hardware that is being used to enhance first-generation interferometers to obtain a ten-fold increase in searchable volume in the timeframe from 2009 to 2011. Construction of a second generation of detectors has begun, which will enable searches over a thousand-fold greater volume than any previous search. These advances are likely to produce regular detections of gravitational waves in less than 10 years from now, opening a new subfield of astronomy and a new arena for studying the physics of space and time. On somewhat longer timescales we expect rapid progress extending the range of gravitational wave studies over a broad band of frequencies.

\section{ACKNOWLEDGMENT}

The author gratefully acknowledges the support of the United States National Science Foundation for the construction and operation of the LIGO Laboratory and the Particle Physics and Astronomy Research Council of the United Kingdom, the Max-Planck-Society and the State of Niedersachsen/Germany for support of the construction and operation of the GEO600 detector. The authors also gratefully acknowledge the support of the research by these agencies and by the Australian Research Council, the Natural Sciences and Engineering Research Council of Canada, the Council of Scientific and Industrial Research of India, the Department of Science and Technology of India, the Spanish Ministerio de Educacion y Ciencia, The National Aeronautics and Space Administration, the John Simon Guggenheim Foundation, the Alexander von Humboldt Foundation, the Leverhulme Trust, the David and Lucile Packard Foundation, the Research Corporation, and the Alfred P. Sloan Foundation.

\section{REFERENCES}

[1] Thorne, K S "Gravitational radiation", in Three hundred years of gravitation, Hawking S W and Israel W (Eds.), Cambridge University Press, Cambridge, England Chapter 9, pp. 330-458, 1987.

[2] A. Abramovici, et al, "The laser interferometer gravitational-wave observatory", Science, 256, 325-333, 1992.

[3] The LIGO Scientific Collaboration: B. Abbott, et al, "Detector description and performance for the first coincidence observations between LIGO and GEO”, Nucl.Instrum.Meth., A517, 154-179, 2004.

[4] A. Abramovici, et al, "Improved sensitivity in a gravitational wave interferometer and implications for LIGO", Phys. Lett. A, 218, 157163, 1996.

[5] P. Fritschel, G. Gonzalez, B. Lantz, P. Saha and M. Zucker, "High power interferometric measurement limited by quantum noise and application to detection of gravitational waves", Phys. Rev. Lett., 80, 3181-3184, 1998.

[6] R.W.P. Drever, J.L. Hall, F.W. Kowalski, J. Hough, G.M. Ford, A.J. Munley and H. Ward, "Laser phase and frequency stabilization using an optical resonator", Appl. Phys. B, 31, 97-105, 1983.

[7] G.A. Kerr, N.A. Robertson, J. Hough and C.N. Man, "The fast frequency stabilization of an argon laser to an optical resonator using and extra-cavity electro-optic modulator", Appl. Phys. B 37, 11-16, 1985.

[8] S. Kawamura, A. Abramovici and M.E. Zucker, "Improved multistage wide band frequency stabilization”, Rev. Sci. Instrum., 68, 223-229, 1997.

[9] D. Shoemaker, P. Fritschel, J. Giaime, N. Christensen and R. Weiss, "Prototype Michelson interferometer with Fabry-Perot cavities", Appl. Opt., 30, 3133-3138, 1991.

[10] M.W. Regehr, F.J. Raab and S.E. Whitcomb, "Demonstration of a power-recycled Michelson interferometer with Fabry-Perot arms by frontal modulation", Opt. Lett., 20, 1507-1509, 1995.

[11] R. Flaminio and H. Heitman, "Longitudinal control of an interferometer for the detection of gravitational waves", Phys. Lett. A, 214, 112-122, 1996.

[12] D. Sigg, N. Mavalvala, J. Giaime, P. Fritschel and D. Shoemaker, "Signal extraction in a power recycled Michelson interferometer with 
Fabry-Perot arm cavities by use of a multiple-carrier frontal modulation scheme", Appl. Opt., 37, 5687-5693, 1998.

[13] J. Camp, L. Sievers, R. Bork and J. Heefner, "Guided lock acquisition in a suspended Fabry-Perot cavity", Opt. Lett., 20, 2463-2465, 1995.

[14] S. Kawamura and M.E. Zucker, "Orientation noise in a Fabry-Perot interferometer gravitational wave detector”, Appl. Opt., 33, 3912-3918, 1994.

[15] N. Mavalvala, D. Sigg and D. Shoemaker, "Experimental test of an alignment sensing scheme for a gravitational wave interferometer", Appl. Opt., 37, 7743-7746, 1998.

[16] D. Sigg and N. Mavalvala, "Principles of calculating the dynamical response of misaligned complex optical interferometers", J. Opt. Soc. Am. A, 17, 1642-1649, 2000.

[17] D. Sigg and R.G. Beausoleil, "Spatiotemporal model of a LIGO interferometer", J. Opt. Soc. Am. A, 16, 1642-1649, 1999.

[18] J. Giaime, P. Saha, D. Shoemaker and L. Sievers, "A passive vibration isolation stack for LIGO: design, modeling and testing", Rev. Sci. Instrum., 67, 208-214, 1996.

[19] R.W.P. Drever, "Interferometric detectors for gravitational radiation", Gravitational Radiation, N. Deruelle and T. Piran, pp 321-328, NorthHolland, Amsterdam, 1983.

[20] B.J. Meers, "Recycling in laser-interferometric gravitational-wave detectors", Phys. Rev. D, 38, 2317-2326, 1988.

[21] R. Weiss, "Electromagnetically coupled broadband gravitational antenna", Q. Prog. Rep. Res. Lab. Electr.MIT, 105, 54, 1972.

[22] J. Vinet, B. Meers, C.N. Man and A. Brillet, "Optimization of longbaseline interferometers for gravitational wave detection", Phys. Rev. D, 38, 433-447, 1988.

[23] T.M. Niebauer, R. Schilling, K. Danzmann, A. Rudiger and W. Winkler, "Nonstationary shot noise and its effect on the sensitivity of interferometers", Phys. Rev. A, 43, 5022-5029, 1991.

[24] T.T. Lyons, M.R. Regehr and F.J. Raab, "Shot noise in gravitational wave detectors with Fabry-Perot arms", Appl. Opt., 39, 6761-6170, 2000.

[25] P.R. Saulson, "Thermal noise in mechanical experiments", Phys. Rev. D, 42, 2437-2345, 1990.

[26] A. Gillespie and F. Raab, "Thermally excited vibrations of the mirrors of laser interferometric gravitational wave detectors", Phys. Rev. D 52 $577-585,1995$.

[27] Y. Levin, "Internal thermal noise in LIGO test masses: A direct approach", Phys. Rev. D, 57, 659-663, 1998.

[28] G.H. Harry, A.M. Gretarsson, P.R. Saulson, S.E. Kittelberger, S.D. Penn, W.J. Startin, S. Rowan, M. Fejer, D.R.M. Crooks, G. Cagnoli, J. Hough and N. Nakagawa, "Thermal noise in interferometric gravitational wave detectors due to dielectric optical coatings", Class. Quant. Grav., 19, 897-917, 2002.

[29] A. Gillespie, "Thermal noise in the initial LIGO interferometers", PhDThesis, California Institute of Technology, Pasadena, 1995.

[30] G. Gonzalez and P.R. Saulson, "Brownian motion of a mass suspended by an anelastic wire", J. Acoust. Soc. Am. 96, pp. 207-212, 1994.

[31] P.R. Saulson, "Terrestrial gravitational noise on a gravitational wave antenna", Phys. Rev. D, 80, pp. 732-736, 1984.

[32] S.A. Hughes and K.S. Thorne, "Seismic gravity-gradient noise in interferometric gravitational-wave detectors", Phys. Rev. D, 58, $122002,1998$.

[33] E. Flanagan and K.S. Thorne, "Noise due to backscatter off baffes, the nearby wall, and objects at the far end of the beam tue; and recommended actions", LIGO Technical Report LIGO-T940063-00-R, 1994, available at http://www.ligo.caltech.edu/docs/T/T940063-00.pdf.

[34] Willke B. "GEO600: status and plans", Class. Quant. Grav. 24, pp. S389-398, 2007.

[35] Acernese F, et al, "Status of the Virgo detector", Class. Quant. Grav. 24, pp. S381-S388, 2007.

[36] Tatsumi, D., "Current status of Japanese detectors", Class. Quant. Grav. 24, pp. S399-S404, 2007.

[37] N. A. Robertson, et al., "Seismic isolation and suspension systems for advanced LIGO", in Gravitational Wave and Particle Detectors, ed. By
J. Hough and G. Sanders, vol. 5500 of Proceedings of SPIE, page 81 2004.

[38] S. Ballmer, et al., "Thermal compensation system description", LIGO Technical Report LIGO-T050064-00-R, 2005, available at http://www.ligo.caltech.edu/docs/T/T050064-00.pdf.

[39] http://www.lsc-group.phys.uwm.edu/ppcomm/Papers.html maintains an updated listing of analysis papers from LIGO.

[40] B. Allen, "The stochastic gravity-wave background: sources and detection.", Proceedings of the Les Houches School on Astrophysical Sources of Gravitational Waves, eds. Jean-Alain Marck and Jean-Pierre Lasota, Cambridge, 1996 (available from arXiv:gr-qc/9604033v3).

[41] T.L. Smith, E. Pierpaoli and M. Kmionkowski, "New Cosmic Microwave Background Constraint to Primordial Gravitational Waves", Phys. Rev. Lett., 97, 021301, 2006.

[42] The LIGO Scientific Collaboration: B. Abbott, et al, "Implications for the Origin of GRB 070201 from LIGO Observations", arXiv:0711.1163v2 [astro-ph],to appear in Ap.J.

[43] The LIGO Scientific Collaboration: B. Abbott, et al, "Beating the spindown limit on gravitational wave emission from the Crab pulsar", arXiv:0805.4758v1 [astro-ph], submitted to Ap.J.Lett.

[44] Advanced LIGO Team, "Advanced LIGO reference design", LIGO technical document LIGO-M060056-10-M, available at http://www.ligo.caltech.edu/docs/M/M060056-10.pdf

[45] J. Baker, et al., "LISA: Probing the universe with gravitational waves", http://www.srl.caltech.edu/lisa/documents/lisa_science_case.pdf and references therein. 